\title{
Microfluidic Devices for Monitoring the Root Morphology of Arabidopsis Thaliana in situ
}

\author{
Lijun Sun, ${ }^{* 1+}$ Lili LIU, ${ }^{* 1}$ Xiangyun LIN, ${ }^{* 2}$ Zhiyi XIA, ${ }^{* 1, * 3}$ Jingli CaO,${ }^{* 1, * 4}$ Shaofu Xu, ${ }^{* 1}$ Haiying Gu, ${ }^{* 2}$ \\ Haibing Y ANG, $^{* 5}$ and Ning BAO ${ }^{* 2}$ \\ *1 School of Life Sciences, Nantong University, 9 Seyuan Rd., Nantong, Jiangsu 226019, China \\ *2 School of Public Health, Nantong University, 9 Seyuan Rd., Nantong, Jiangsu 226019, China \\ *3 School of Life Sciences, Central South University, 100 Xiangya Rd., Changsha, Hunan 410078, China \\ *4 School of Basic Medical Sciences, Fudan University, 220 Handan Rd., Shanghai 200433, China \\ *5 South China Botanical Garden, Chinese Academy of Sciences, 723 Xingke Rd, Guangzhou 510650, China
}

\begin{abstract}
Plant roots play critical roles in absorbing nutrients for the growth and development of plants as well as adapting different environments. Currently, there is no satisfactory way to track dynamic information when studying roots at the high temporal and spatial resolution. Herein, a simple microfluidic device with crossed microchannels was utilized for a microscopic investigation of Arabidopsis thaliana roots in situ. Our experimental results showed that the microfluidic system combined with a microscope could be conveniently utilized for the quantification of primary roots and root hairs with a change of micrometers within a time of minutes. Using the same approach, the influences of high salinity stress could also be investigated on different parts of roots, including the root cap, meristematic zone, elongation zone, mature zone, and root hairs. More importantly, the growth of roots and root hairs could be quantified and compared in a solution of abscisic acid and indole-3-acetic acid, respectively. Our study suggested that the microfluidic system could become a powerful tool for the quantitative investigation of Arabidopsis thaliana roots.
\end{abstract}

Keywords Microfluidic system, root morphology, in situ, abscisic acid, indole-3-acetic acid

(Received August 4, 2020; Accepted October 9, 2020; Advance Publication Released Online by J-STAGE October 23, 2020)

\section{Introduction}

Plant roots are critical for not only absorbing nutrients for growth and the development of plants, but also adapting different environments, or various stresses. ${ }^{1-3}$ Concerning plant morphology, normally the plant root includes the root cap, meristematic zone, elongation zone, mature zone, and root hair. ${ }^{2}$ Although significant progress has been made on understanding the mechanisms of plant roots interaction under biotic and abiotic stresses, it is still not very clear for concerning responses of different zones of roots under biotic and abiotic stresses at high resolutions of time and space. This is because it is not easy to observe microscale changes of flexible plant roots in complex soil pots or agarose plates. It would be even more difficult when other morphologic parameters, including the growth speed and real-time cellular behaviors (e.g., cell division, elongation, and interactions) are involved.

To date, the microfluidic system has recently become a potential platform for studying plants. ${ }^{4,5}$ It has been demonstrated that microfluidic devices could not only be used for studying roots of Arabidopsis thaliana at high temporal and spatial resolutions but could also allow seamlessly changing the growth conditions..$^{6-8}$ The platform of "plant on a chip" was developed

L. S., L. L. and X. L. contributed equally to this work.

$\dagger$ To whom correspondence should be addressed.

E-mail: slj.1226@163.com (L. S.), ningbao@ntu.edu.cn (N. B.) for studying influences of 2,4-dichlorophenoxyacetic acid $(2,4-$ D), a synthetic derivative of auxin, and its inhibitor ( $N-1$ naphthylphthalamic acid) on development in Arabidopsis thaliana roots. ${ }^{8}$ The concept of RootChip was proposed by Grossmann's group through monitoring time-resolved growth and cytosolic sugar levels at the subcellular resolution in Arabidopsis thaliana roots using a genetically encoded fluorescent marker for glucose and galactose. ${ }^{7}$ Coupled with the computational technique, the microfluidic device had also been used for high-throughput imaging of gene expression in Arabidopsis thaliana roots. ${ }^{6}$ More recently, microfluidic systems have been utilized for studying the interaction between plant roots and microbes based on microscopic imaging. ${ }^{9}$ Those investigations demonstrated the potential of microfluidic systems for studying plant growth.

In this study, microfluidic devices with simply crossed microchannels were utilized for a quantitative investigation of Arabidopsis thaliana roots under abiotic stresses. Compared with the single channel in previous reports, ${ }^{6-9}$ the crossed microchannels of our study can supply more opportunities for Arabidopsis roots to grow with a certain direction in the channel. Our results showed that microfluidic chips with crossed microchannels could be used to quantitatively monitor the growth and development of roots and root hairs in situ in real-time. This study suggested that the microfluidic system could become an alternative tool for a real-time study of plant roots in situ. 

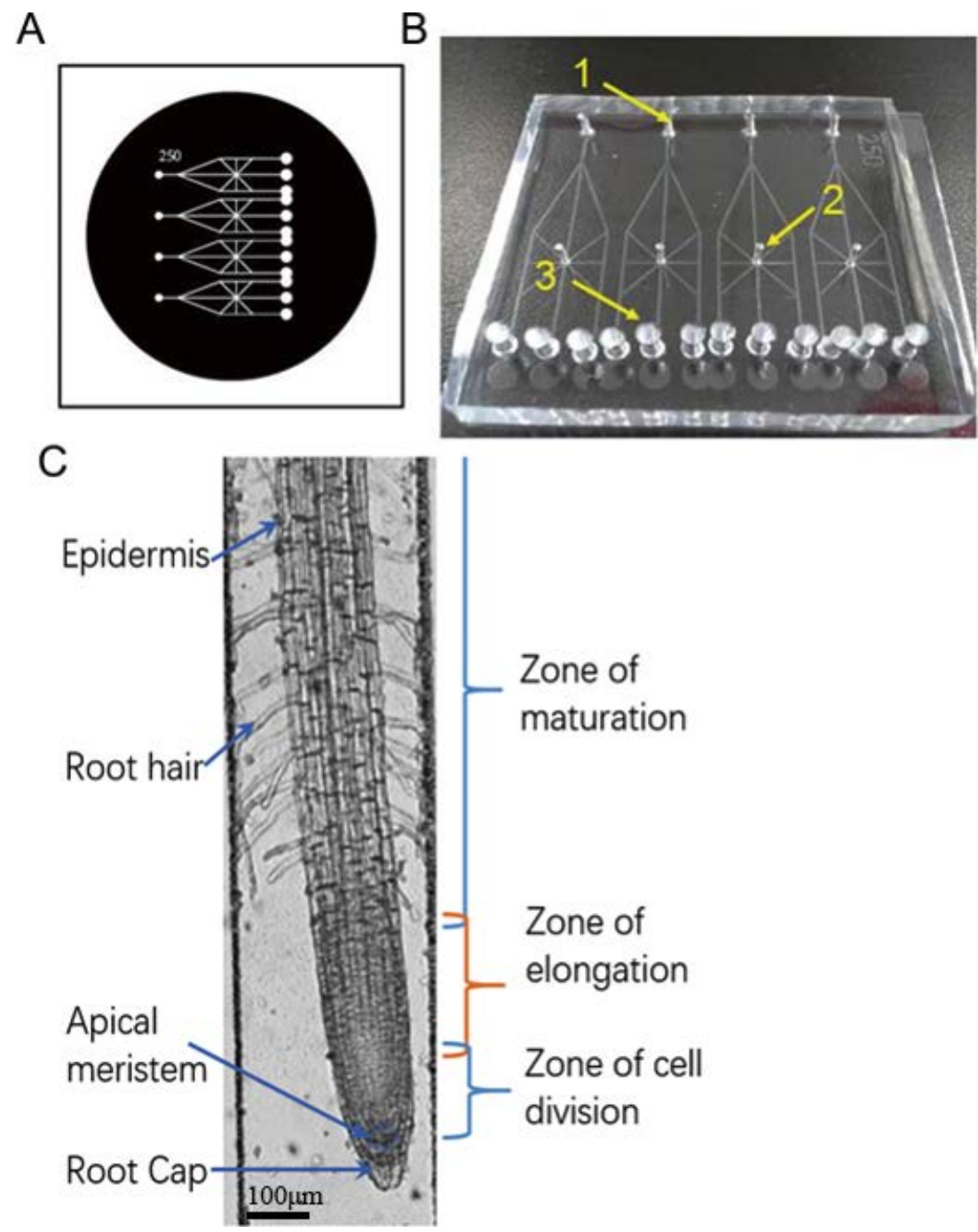

Fig. 1 Construction of transparency film and microchannels. (A) Transparency film of the microchannel structure with simply crossed microchannels, (B) Simply crossed microchannels with diameters of 1.5, 1.0, and $2.5 \mathrm{~mm}$ holes for the liquid flow into the microchannels (1), seed implantation (2), and liquid flow out (3). (C) Structure of a root tip of Arabidopsis thaliana which was cultured in the microchannel. Scale bar, $100 \mu \mathrm{m}$.

\section{Experimental}

\section{Reagents and materials}

SU-8 GM1075 photoresist was purchased from the Gersteltec (Vaud, Switzerland). Single silicon chips with a diameter of $76 \mathrm{~mm}$, a thickness of $365-380 \mu \mathrm{m}$, and a resistivity of $<0.02 \Omega \mathrm{cm}$ were purchased from Suzhou double metal materials Co., Ltd. (Suzhou, China). PDMS silgard184 was bought from Dow Corning (Michigan, USA). A Harris-CoreTM punch with diameters of $1,1.5$, and $2.5 \mathrm{~mm}$ were purchased from Beijing Kerry biological technology Co., Ltd. (Beijing, China). One ml syringes were obtained from Nantong's biological experiment equipment Co., Ltd. (Nantong, China). Indole-3-acetic acid and abscisic acid were obtained from Sigma (Missouri, USA). Other reagents were of analytical grade. All solutions were prepared with twice-distilled water and stored at $4^{\circ} \mathrm{C}$.

\section{Microchip fabrication}

PDMS-based microfluidic devices were fabricated according to the standard soft lithography, as reported previously. ${ }^{10}$ Briefly, a microchannel structure with simply crossed microchannels was designed using illustrator software (Adobe, San Jose, CA), and then printed out on transparency film (Fig. 1A). The SU-8 GM1075 was coated on a silica wafer using a spin coater (MYCRO WS-650Mz-23NPP) from Laurell (Maryland, USA). The transparency was used as a photomask for coating photoresist silicon, which was exposed to EXFO OmniCure $^{\circledR}$ S1000 ultraviolet point light (Shanghai, China) to form a silicon mold having a microfluidic structure. The PDMS Sylgard 184 mixture (monomer and curing agent with a ratio of 10:1) was poured onto the mold and solidified. A PDMS chip with a microfluidic structure and a glass slide were cleaned through PDC-32 GB oxygen plasma and pasted together to form Microchip (New York, USA). Thus, a microfluidic platform with simply crossed microchannels was performed (Fig. 1B). Punchers with diameters of 1.5, 1.0, and $2.5 \mathrm{~mm}$ were used to punch holes, which were used for liquid flow into 

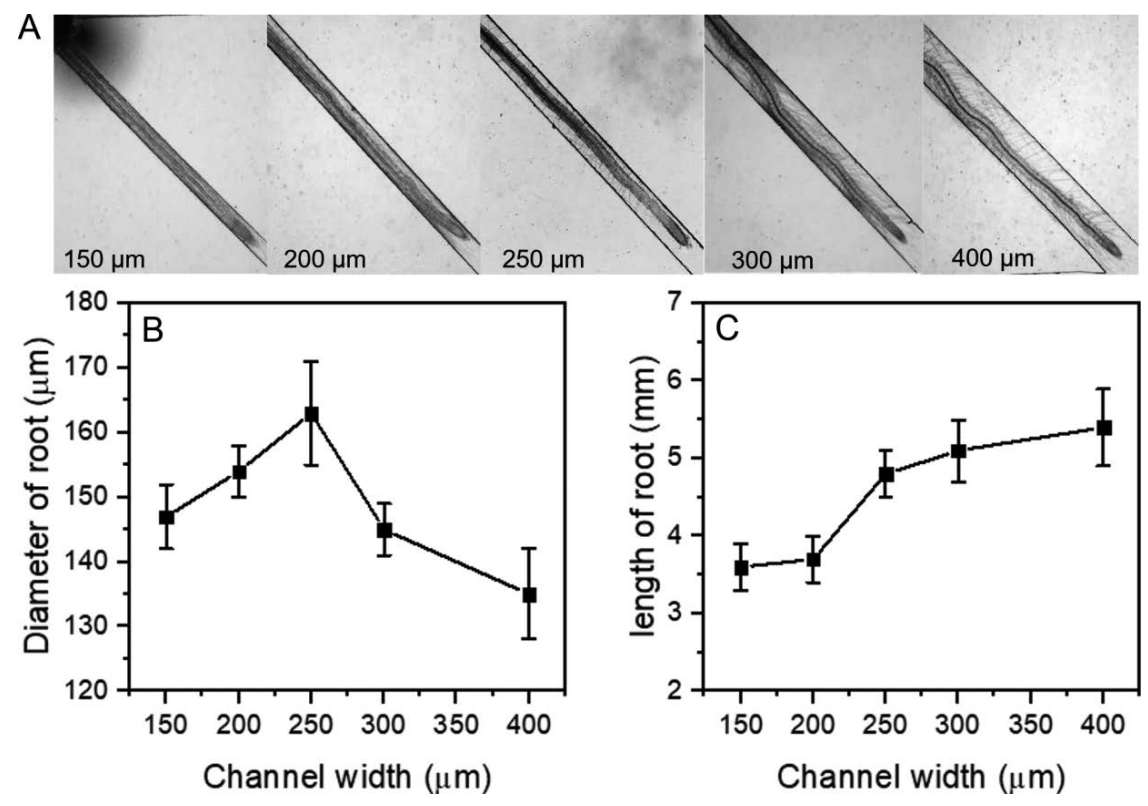

Fig. 2 The influence of the microchannel width on the diameters and lengths of the Arabidopsis thaliana roots. (A) Growth status of Arabidopsis in the microchannels. Diameters (B) and lengths (C) of the Arabidopsis thaliana roots in microchannels with different diameters on the seventh day $(n=4)$.

the microchannels (1), seed implantation (2), and liquid outflow (3). Teflon tubing was connected to the inlets of the channels for transporting solution by Langer LSP02-1B double-channel injection pump (Nanjing, China). The flow rate of the solution was $0.5 \mu \mathrm{L} / \mathrm{min}$.

\section{Growth of Arabidopsis in the microfluidic device}

Wild type Arabidopsis thaliana ecotype Columbia (Col-0) seeds were surface sterilized and preincubated at $4^{\circ} \mathrm{C}$ for $48 \mathrm{~h}$ in the dark. The seeds were then placed in microfluidic channels for germination, and grew in an incubator with a 16-h light/8-h dark photoperiod at $25^{\circ} \mathrm{C}$ for $6-7$ days. There were two highsalinity treatments: Salinity-I, in which the seeds were germinated in the micro-channel in distilled water for $48 \mathrm{~h}$ and then treated with a $0.15 \mathrm{M} \mathrm{NaCl}$ solution on the third day, and Salinity-II, in which the seeds were germinated in the microchannel in a $0.15 \mathrm{M} \mathrm{NaCl}$ solution from the first day. For treating with plant hormones, Abscisic acid (ABA, $4.0 \mu \mathrm{M})$ or Indole-3-acetic acid (IAA $1.0 \mathrm{nM}$ ) was used to study the influence on the development of Arabidopsis. Double-distilled water was used as the control of all experiments.

\section{Image acquisition}

Images of the roots of Arabidopsis were taken on using a Leica DMI 3000b fluorescent inverted microscope (Wetzlar, Germany). The data were extracted using the software of ImageJ from the images. All of the results were obtained under the same experimental conditions. Normally the data were calculated based on results of four parallel experiments.

\section{Results and Discussion}

Being exposed in the soil (sometimes, the air), the roots of plants could not only absorb water and nutrition, but could also respond to stresses. Although it has been reported that mechanical stress from soil could either limit or stimulate the growth of roots, no quantitative data had been reported.
Figure 1C illustrates the structure of a root tip of Arabidopsis thaliana that was cultured in a PDMS microchannel. Every part of the roots could be observed. At the end of each root is the root cap, and above it is the zone of cell division. Between them is the section of the apical meristem. Above the zone of cell division are the zone of elongation and the zone of maturation. It needs to be emphasized that herein the PDMS microchannels attached on the glass slide were used for only observing the growth of the plant roots in the microchannels. If it is necessary to take the cultured plants outside the microchannel, microfluidic devices might be fabricated by applying adhesive tape to combine the cover slip and the glass slide.

Figure 2 illustrates that microfluidic channels with various channel width offered a perfect platform for a quantitative study of the mechanical stresses on the growth of Arabidopsis thaliana roots. The growth status, including the variation for roots elongation and diameter were observed for one week. It could be found that the maximum root diameter could reach $163 \mu \mathrm{m}$ in a $250 \mu \mathrm{m}$ micro-channel while the root width can reach $146 \mu \mathrm{m}$ in a $150 \mu \mathrm{m}$ micro-channel, almost filling the entire channel. Meanwhile, the length of the roots gradually increased with the increase of the channel widths. The meristem and elongation of root hairs were severely hindered in the narrower channels (150 and $200 \mu \mathrm{m})$. In microchannels with widths from 250 to $400 \mu \mathrm{m}$, the roots grew well based on observations of the root elongation and root hairs. It should be emphasized that the channel depth was $60 \mu \mathrm{m}$, which might be a factor concerning the limits of the root and root hairs growth. In our following experiment, microchannels with a width of $250 \mu \mathrm{m}$ were used for growing Arabidopsis roots.

Figure 3 illustrates the increase in the length of one Arabidopsis root hair within $120 \mathrm{~min}$ in the microchannel channels. It is well known that root hairs are single-cell tubular extensions of root epidermal cells, and contribute to the efficient acquisition of water and nutrients. ${ }^{3,11}$ Root hairs could interact with the environments (mainly, the soil) by enlarging the surface area of the root. Thus, the development of root hairs is strongly 

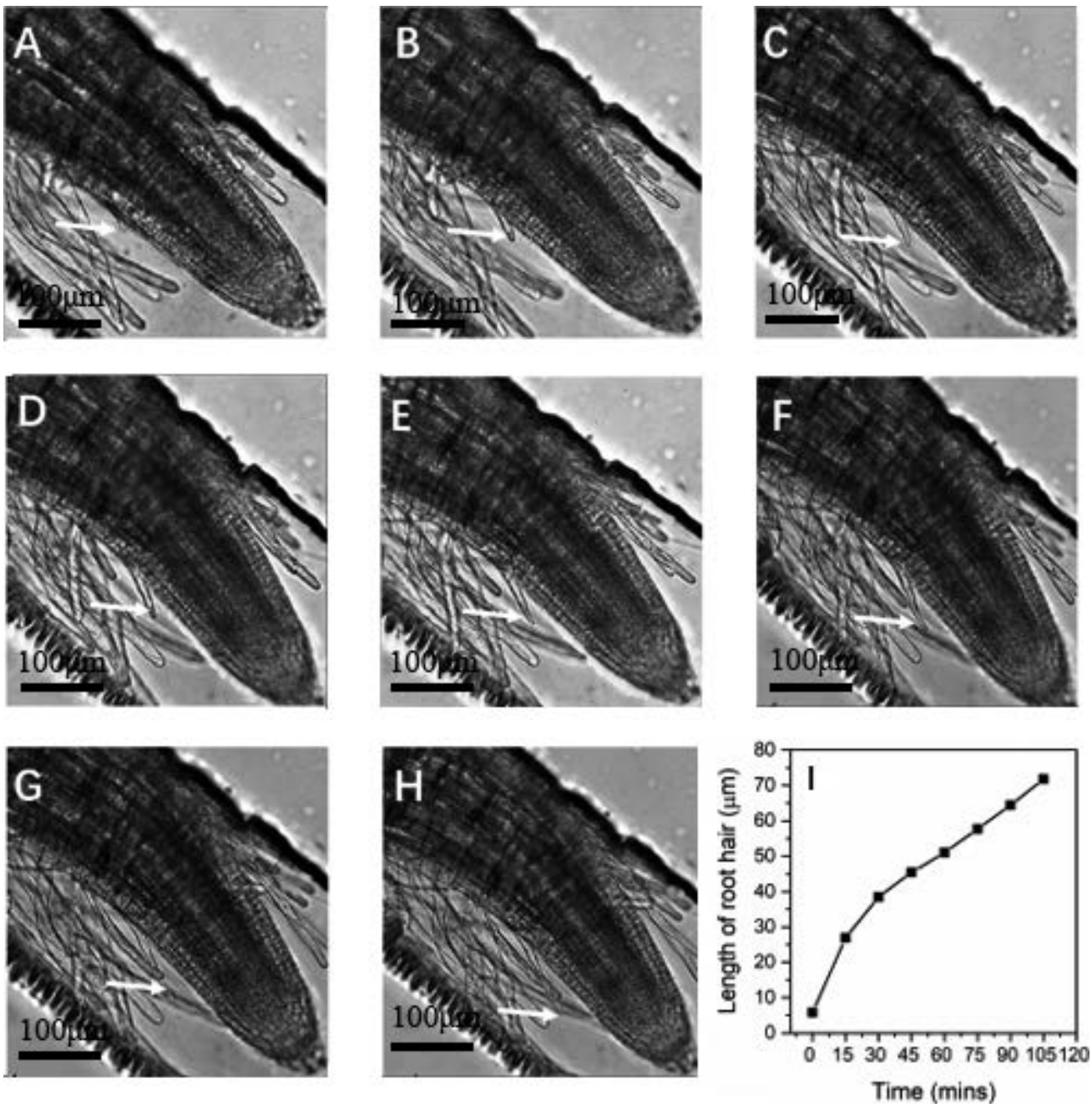

Fig. 3 Growth status and dynamic changes of an Arabidopsis root hair in the microchannel. A to $\mathrm{H}$ present the elongation process of the root hair. The arrows indicate specific root hair. I presents the elongation speeds of the root hair. Scale bar, $100 \mu \mathrm{m}$.

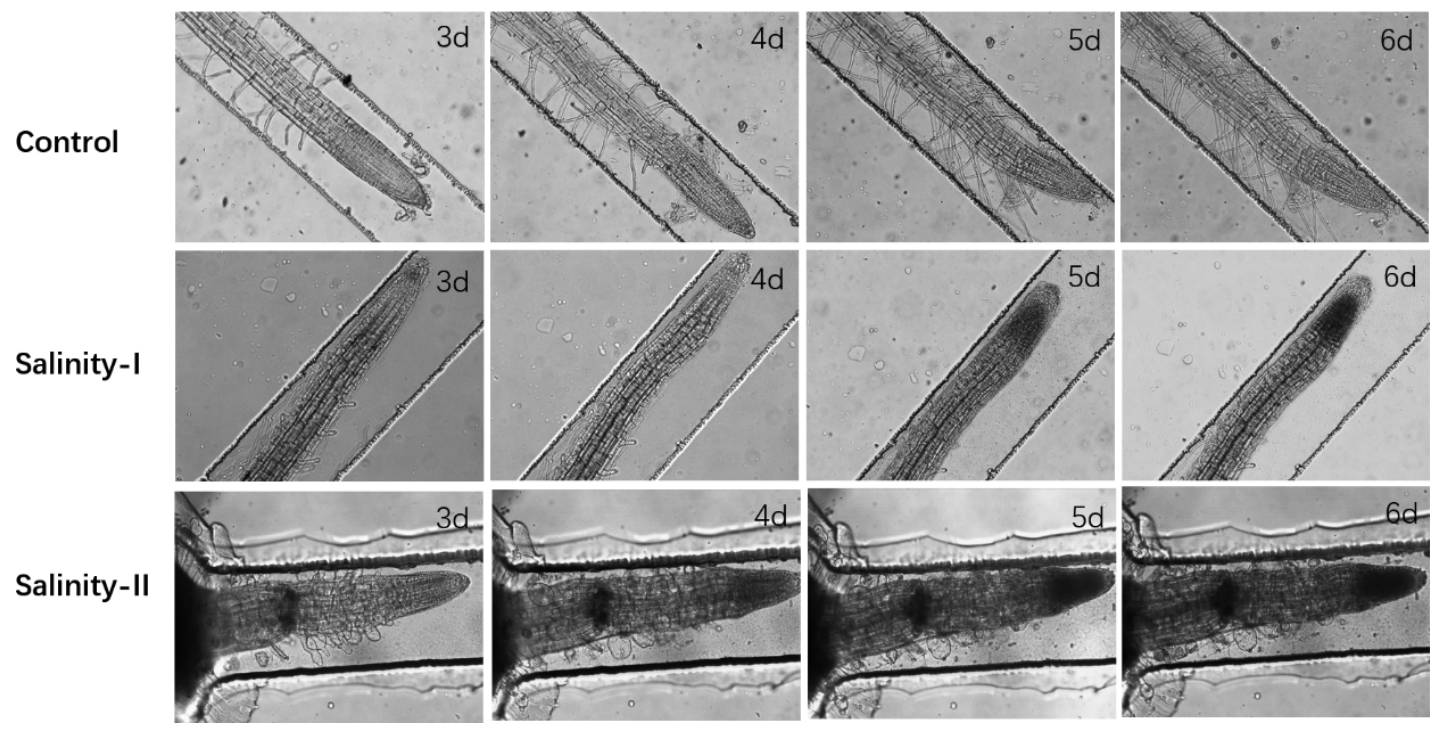

Fig. 4 Micromorphology of Arabidopsis thaliana roots in microfluidic channels in the control, Salinity-I and Salinity-II. The control is that the seeds were germinated and grew in twice-distilled water. Salinity-I is that the seeds were germinated in the micro-channel within the distilled water for $48 \mathrm{~h}$, and then treated with a $0.15 \mathrm{M} \mathrm{NaCl}$ solution on the third day, Salinity-II is that the seeds were germinated in the micro-channel within the $0.15 \mathrm{M} \mathrm{NaCl}$ solution on the first day. Scale bar, $100 \mu \mathrm{m}$. 
A

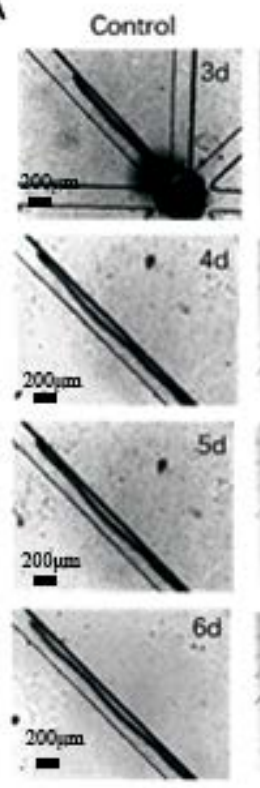

ABA
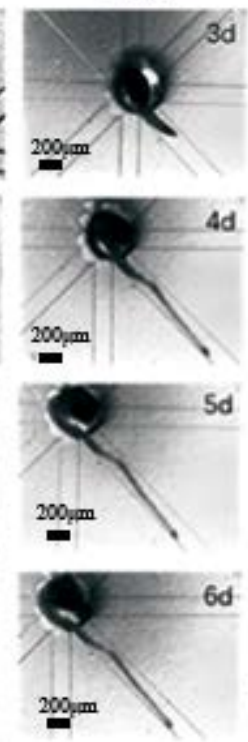

IAA
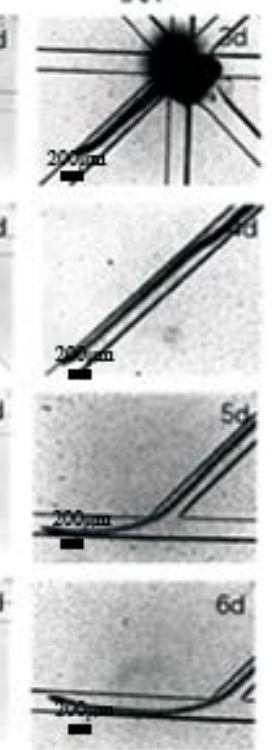

B

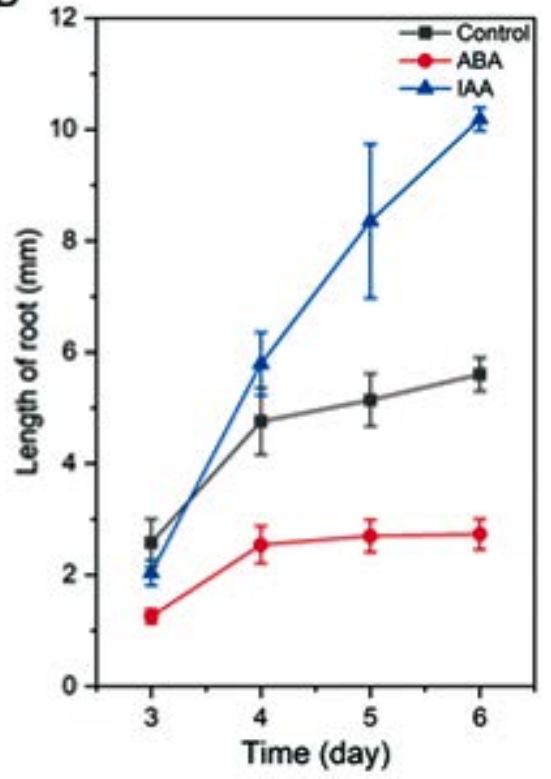

Fig. 5 A) Micromorphology of Arabidopsis thaliana roots response on ABA and IAA in the microfluidic channels. B) Effect of ABA and IAA on the elongation of Arabidopsis' main root $(n=4)$. Arabidopsis were cultured in double-distilled water (the control), $4.0 \mu \mathrm{M} \mathrm{ABA}$, or 1.0 nM IAA. Scale bar, $200 \mu \mathrm{m}$.
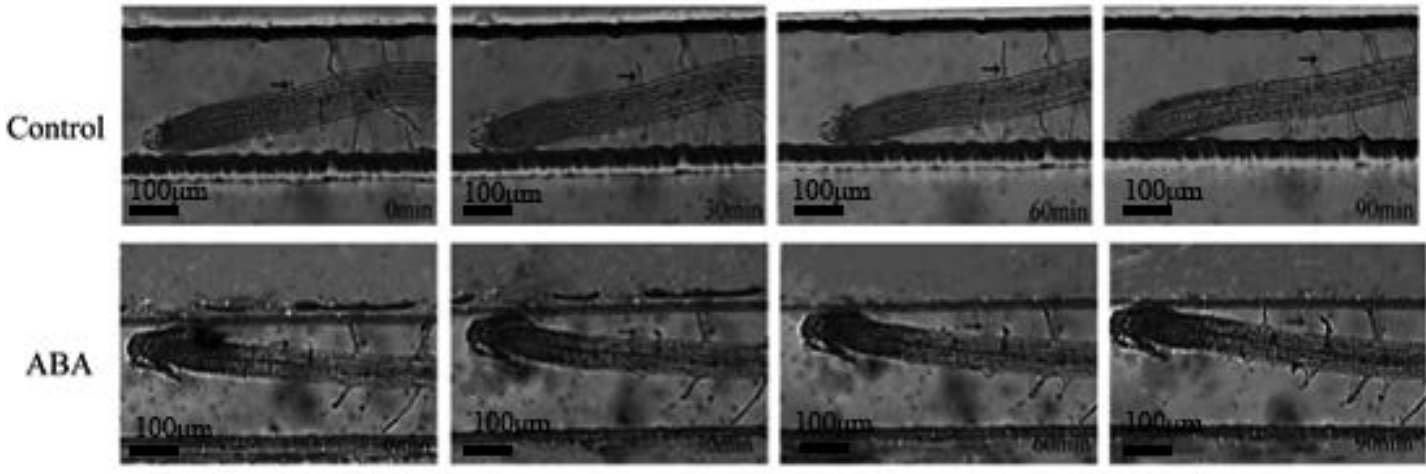

ABA
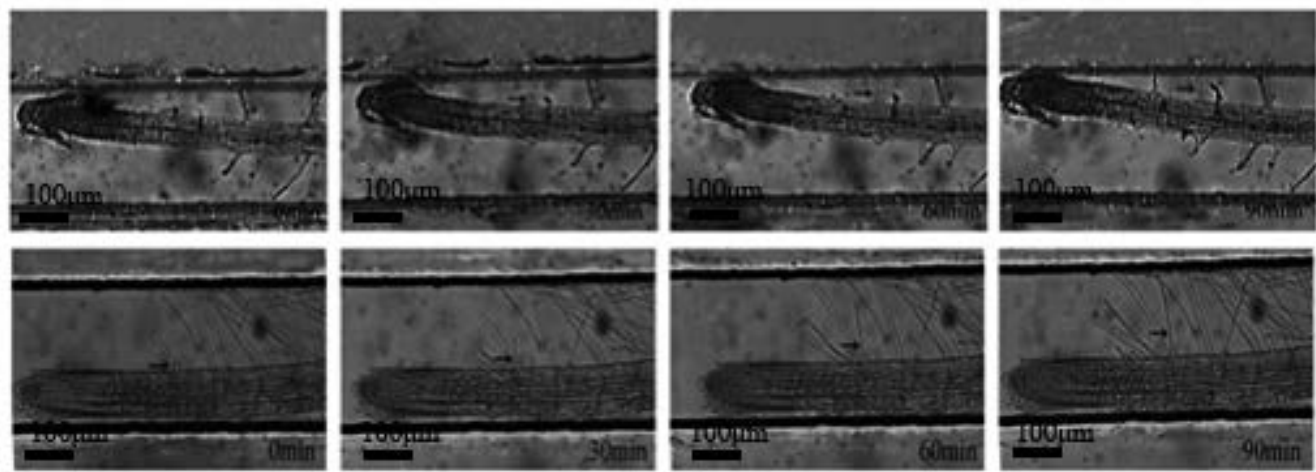

Fig. 6 Micromorphology of Arabidopsis thaliana root hairs in the control and the solution consisting of ABA and IAA in the microfluidic channels (magnification of 200, the arrows indicate the specific root hair). Arabidopsis were cultured in double-distilled water (the control), $4.0 \mu \mathrm{M} \mathrm{ABA}$, or $1.0 \mathrm{nM}$ IAA. Scale bar, $200 \mu \mathrm{m}$.

influenced by the surrounding conditions, which might be one of the important mechanisms of the environmental adaptation of plants. ${ }^{3,11}$ In the microchannels, it could be observed that within $120 \mathrm{~min}$ the length of the root hair increased from 6 to $74 \mu \mathrm{m}$ without stopping. The elongation speed of the root hair was faster at the very beginning, but then decreased. It should be noted that distilled water was used in our investigation, which could influence the growth of the root hairs. Such results implied that microfluidic systems offered a suitable tool for the quantitative analysis of root hairs at the level of micrometers within a time of minutes

The microfluidic devices were then used to study the influences of high-salinity stress on the roots of Arabidopsis thaliana roots. The stress of salinity is one of the major abiotic factors that limit plant growth and productivity. ${ }^{12,13}$ This is because the roots are the first plant organ to sense and respond to the salinity. ${ }^{14,15}$ Figure 4 shows that the roots grew well with well-developed root hairs in the control group (without the high-salinity stress). In comparison, the roots of Arabidopsis thaliana grew more slowly under the stresses of the high salinity. 

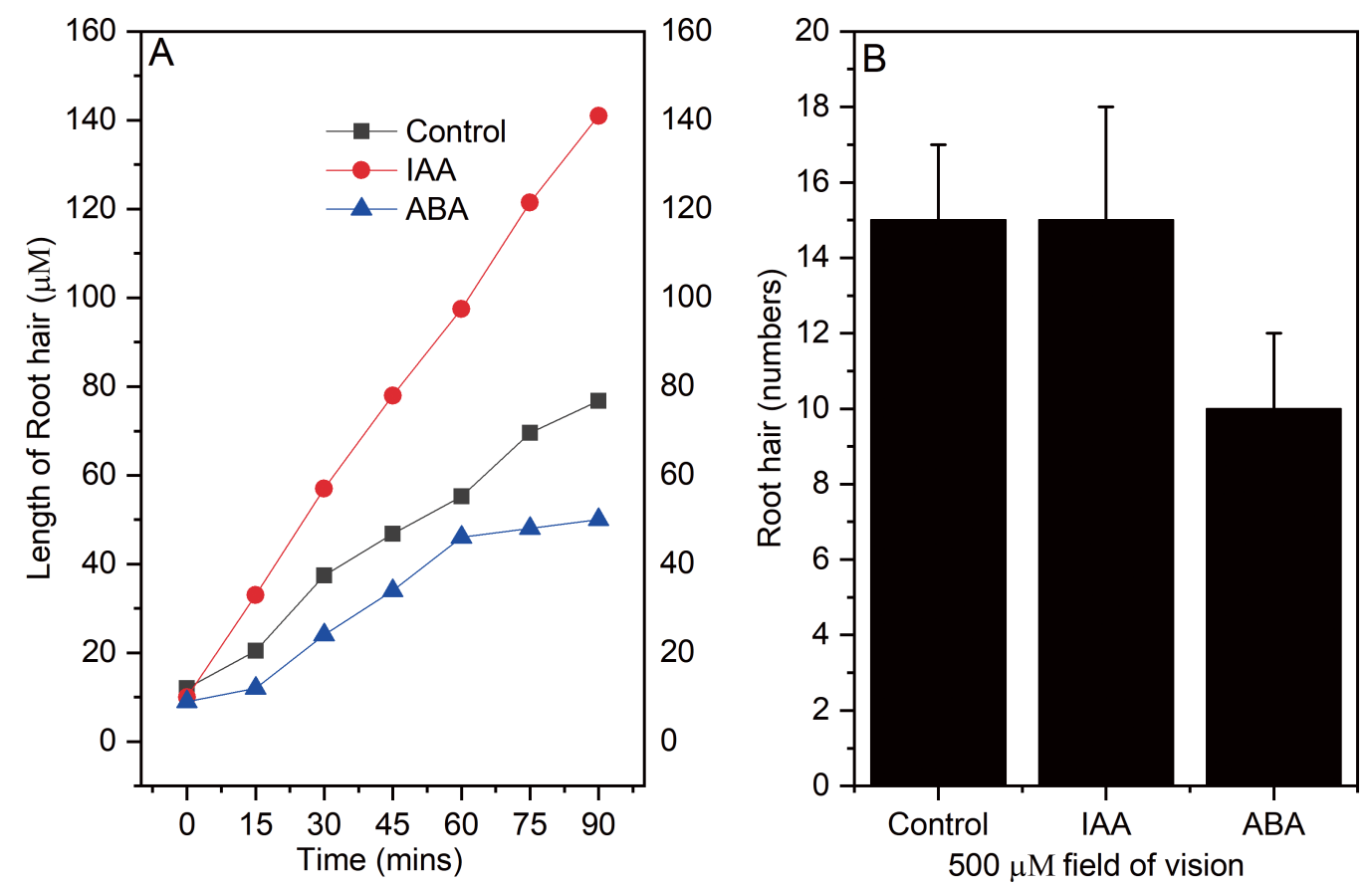

Fig. 7 A) Elongation speeds of a specific root hair in the control and the solution consisting of. ABA and IAA. B) The numbers of root hair in the $500 \mu \mathrm{m}$ field of vision in the control and the solution consisting of ABA and IAA $(n=4)$.

In addition, it could be found that the color in the areas of cell differentiation and cell division became darker with passing time, especially under the stress of higher salinity, implying the increased density and the suppressed growing speed of the cells in these zones. The enlarged root caps could also be observed under high-salinity stress, which might be ascribed to the protective function of the roots. The number and length of root hairs significantly decreased, suggesting the inhibition effect of high-salinity stress. Some vesicular root hairs could be observed under the higher salinity (Salinity-II). Such results showed that microfluidic devices could be used to monitor phenotypic changes of root structure in a continuous and non-invasive way.

$\mathrm{ABA}$ and IAA are two kinds of major plant hormones that could control many cellular processes, including the regulation of primary root growth in plants. ${ }^{16,17}$ ABA and IAA can exert promotive or inhibitive effects on the growth of the primary root, depending on their concentrations. They could stimulate the formation of roots at a lower concentration, while having an inhibitory effect at higher concentration. ${ }^{16,17}$ Figure 5 shows the effects of ABA and IAA on the development of Arabidopsis thaliana roots in microfluidic channels. It could be found that ABA $(4.0 \mu \mathrm{M})$ significantly inhibited elongation of the main roots, while IAA $(1.0 \mathrm{nM})$ significantly promoted elongation of the main roots. More importantly, using the microfluidic devices, it can be found from Fig. 5B that the changes in the root length could be quantified, which could be used for studying the influences of the biotic stresses on root development.

Figure 6 shows the influences of external ABA and IAA on the growth of root hairs. It could be found that ABA dramatically inhibited the elongation and numbers of Arabidopsis root hairs. On the contrary, IAA significantly promoted the elongation of Arabidopsis root hairs. Figure 7 illustrates quantitative changes in the length and number of root hairs. It seems that the promotion impact of IAA was more than the inhibition influence of $\mathrm{ABA}$ on the length of root hairs. Besides, IAA did not change the number of root hairs very much, but ABA decreased the number of root hairs. Rymen reported that the ABA can suppress the growth of root hairs via the OBP4 transcriptional regulator. ${ }^{18}$ Auxin had been reported to promote root hair elongation in Arabidopsis at low concentraiton. ${ }^{19,20}$ Microfluidic systems have been reportedly coupled with imaging, genetic engineering and computational modeling for studying root growth influenced by auxin. ${ }^{21}$ Our results provided quantitative evidence for the influences of IAA and $\mathrm{ABA}$ on the growth of roots and root hairs.

\section{Conclusions}

In this study, microfluidic devices with simply crossed microchannels were used for a quantitative study of the growth and development of Arabidopsis thaliana roots. Our results showed that the in situ growth and development of Arabidopsis thaliana roots could be tracked at the level of minutes and micrometers. The microfluidic systems integrated with a microscope could be used to perform quantitative analysis of the growth of roots and root hairs not only under the stress of high salinity, but also in external IAA or ABA. It could be envisioned that such analytical data could help us to deeply understand the dynamic changes of plant roots. This study might offer an alternative for investigating the mechanism between the plant roots and environment conditions with quantity rather than quality.

\section{Acknowledgements}

The authors appreciate financial support from the National Natural Science Foundation of China (Nos. 31770399, 21375066, and 31400315), the Natural Science Foundation of Jiangsu 
Province (No. BK20130389), Qing Lan Project of Jiangsu Province, Six talent peaks project in Jiangsu Province (No. SWYY-061), and science and technology innovation project of Jiangsu Province (No. KYCX17-1908).

\section{References}

1. D. Bhardwaj, A. Medici, A. Gojon, B. Lacombe, and N. Tuteja, Plant Signaling Behav., 2015, 10, e1049791.

2. C. D. Canto, M. Simonin, E. King, L. Moulin, M. J. Bennett, G. Castrillo, and L. Laplaze, Plant J., 2020, 102, 951.

3. K. Vissenberg, N. Claeijs, D. Balcerowicz, and S. Schoenaers, J. Exp. Bot., 2020, 71, 2412.

4. T. Prangemeier, F. X. Lehr, R. M. Schoeman, and H. Koeppl, Curr. Opin. Biotechnol., 2020, 63, 167.

5. A. Sanati Nezhad, Lab Chip, 2014, 14, 3262.

6. W. Busch, B. T. Moore, B. Martsberger, D. L. Mace, R. W. Twigg, J. Jung, I. Pruteanu-Malinici, S. J. Kennedy, G. K. Fricke, R. L. Clark, U. Ohler, and P. N. Benfey, Nat. Methods, 2012, 9, 1101.

7. G. Grossmann, W. J. Guo, D. W. Ehrhardt, W. B. Frommer, R. V. Sit, S. R. Quake, and M. Meier, Plant Cell, 2011, 23, 4234.

8. M. Meier, E. M. Lucchetta, and R. F. Ismagilov, Lab Chip, 2010, 10, 2147.

9. M. F. Noirot-Gros, S. V. Shinde, C. Akins, J. L. Johnson, S.
Zerbs, R. Wilton, K. M. Kemner, P. Noirot, and G. Babnigg, Front. Plant Sci., 2020, 11, 408.

10. P. Kim, K. W. Kwon, M. C. Park, S. H. Lee, S. M. Kim. and K. Y. Suh, Biochip J., 2008, 2, 1.

11. M. Shibata and K. Sugimoto, J. Plant Res., 2019, 132, 301.

12. W. J. Liang, X. L. Ma, P. Wan, and L. Y. Liu, Biochem. Biophys. Res. Commun., 2018, 495, 286.

13. Y. Q. Yang and Y. Guo, J. Integr. Plant Biol., 2018, 60, 796.

14. L. Duan, J. Sebastian, and J. R. Dinneny, "Plant Cell Expansion: Methods and Protocols", ed. J. M. Estevez, 2015, Humana Press.

15. S. Shabala, H. H. Wu, and J. Bose, Plant Sci., 2015, 241, 109.

16. D. Olatunji, D. Geelen, and I. Verstraeten, Int. J. Mol. Sci., 2017, 18.

17. L. R. Sun, Y. B. Wang, S. B. He, and F. S. Hao, Plant Signaling Behav., 2018, 13, e1500069.

18. B. Rymen, A. Kawamura, S. Schafer, C. Breuer, A. Iwase, M. Shibata, M. Ikeda, N. Mitsuda, C. Koncz, M. OhmeTakagi, M. Matsui, and K. Sugimoto, Plant Physiol., 2017, $173,1750$.

19. R. J. Pitts, A. Cernac, and M. Estelle, Plant J., 1998, 16, 553.

20. K. Okada and Y. Shimura, Science, 1990, 250, 274.

21. M. Fendrych, M. Akhmanova, J. Merrin,M. Glanc, S. Hagihara, K. Takahashi, N. Uchida, K.U. Torii, and J. Friml, Nat. Plants, 2018, 4, 453. 